\title{
Perfil de triacilgliceroles y porcentaje de ácido palmítico en la posición sn-2 en sustitutos de leche materna
}

\author{
Profile of triacylglycerols and percentage of palmitic acid at the \\ sn-2 in breast milk substitutes
}

\author{
Dr. Horacio F. González $z^{a}$ Ing. Dimas Vicentin ${ }^{b}$, Ing. Osvaldo Giumelli, \\ Lic. Miguel Vazzano y Dr. Marcelo Tavella
}

a. IDIP - Instituto de Desarrollo e Investigaciones Pediátricas "Prof. Dr. Fernando Viteri", Hospital de Niños de La Plata. MS/CIC-PBA

b. Innovación, Desarrollo e Investigación, SanCor Cooperativas Unidas Limitadas, Sunchales, Santa Fe.

c. PROPIA (Programa de Prevención del Infarto en Argentina), INIBIOLP - Instituto de Investigaciones Bioquímicas de La Plata (UNLPCONICET La Plata), Facultad de Ciencias Médicas, UNLP.

Correspondencia: Dr. Horacio F. González: horaciofgonzalez@ gmail.com

Conflicto de intereses: El Ing. Dimas Vicentin, el Ing. Osvaldo Giumelli y el Lic. Miguel Vazzano reciben honorarios de Innovación, Investigación y Desarrollo de SanCor C.U.L. El Dr. Horacio F. González mantiene una relación contractual con Innovación, Investigación y Desarrollo de SanCor C.U.L.

Recibido: 7-10-2011 Aceptado: 6-1-2012

\section{RESUMEN}

El objetivo del presente trabajo es analizar el porcentaje de ácido palmítico en la posición sn-2 de los triacilgliceroles en sustitutos de la leche materna. Se analizaron 6 productos del mercado argentino que se presentan como sustitutos de la leche materna durante el primer semestre de vida: 2 fórmulas con aceites vegetales como fuente básica de lípidos (F1 y F2); 1 fórmula con grasa láctea como base y aceites vegetales (F3); 1 fórmula con lípidos estructurados y aceites vegetales (F4); 2 fórmulas para prematuros, una con grasa láctea como base y otros aceites (F5) y otra con aceites vegetales (F6). Los resultados muestran que F1, F2, F3 y F4 presentaron $14,6 \%$, $14,8 \%, 48,1 \%$ y $44,5 \%$ del ácido palmítico en la posición sn-2, respectivamente. Por su parte, las fórmulas F5 y F6 presentaron 49,5\% y 14,6\% del ácido palmítico en la posición sn-2. Las fórmulas con base láctea presentaron la mayor concentración de ácido palmítico en la posición sn-2. Palabras clave: ácido palmítico, triacilgliceroles, posición sn-2, fórmulas lácteas, lactantes.

\section{SUMMARY}

The aim of this study was to analyze the percentage of palmitic acid at sn-2 position on triacylglycerols in infant formulas. We studied 6 infant formulas in the Argentinean market that are used as breast-milk substitutes during the first six months after delivery: 2 formulas with vegetable oils as basic source of lipids (F1, F2); 1 formula with milk fat and vegetable oils (F3); 1 formula with structured lipids and vegetable oils (F4); 2 formulas for pre-term infants, one with milk fat and other oils (F5), and the other with vegetable oils (F6). Results showed that F1, F2, F3 and F4 presented $14.6 \%, 14.8 \%, 48.1 \%$ and $44.5 \%$, respectively, of palmitic acid at sn- 2 position, whereas formulas F5 and F6 had $49.5 \%$ and $14.6 \%$, respectively, of palmitic acid at sn- 2 position. Milk-based infant formulas had the highest concentration of palmitic acid at sn-2 position. Key words: palmitic acid, triacylglicerols, sn-2 position, milk formula, infant.

http://dx.doi.org/10.5546/aap.2012.227

\section{INTRODUCCIÓN}

Durante las últimas décadas se ha mostrado un interés creciente en la composición de los ácidos grasos de los sustitutos de leche materna o fórmulas preparadas para lactantes nacidos a término y pretérmino que, por alguna razón, han debido discontinuar la lactancia materna o deben complementarla por indicación médica.

El objetivo en el desarrollo de los sustitutos de la leche materna ha sido producir fórmulas con contenido de grasa, composición y estructura, similares a los que se encuentran en la leche materna. ${ }^{1,2} \mathrm{El}$ uso de diferentes grasas y aceites vegetales ha permitido acercarse a la composición general de las grasas presentes en la leche materna, pero no se ha podido imitar la estructura de los triacilgliceroles. ${ }^{2}$ La absorción de las grasas de la leche materna es superior a la absorbida por los lactantes alimentados con fórmulas infantiles. Una de las razones es que, a pesar de la similitud del perfil lipídico, la estructura de los triacilgliceroles de la leche materna es diferente.

El ácido palmítico (ácido hexadecanoico) es un componente importante de la leche materna; representa alrededor del $25 \%$ de los lípidos en su composición, de los cuales un $60-85 \%$ se encuentra en la posición 2 del triacilglicerol. ${ }^{2-4}$

El agregado de aceite de palma (alto contenido de ácido palmítico) permite lograr una formulación más cercana a la composición de lípidos de la leche materna que la que se obtiene con fórmulas sin dicho aceite. ${ }^{2-4} \mathrm{Sin}$ embargo, uno de los aspectos más críticos de las formulaciones en cuanto a emular a la leche materna es la biodisponibilidad de los nutrientes, en este 
caso particular, de los ácidos grasos.-6

Existen diferencias significativas en la distribución posicional de los ácidos grasos (estereoisomería) en las moléculas de los triacilgliceroles, que provocan diferencias en la absorción de grasa y calcio. La mayor parte del ácido palmítico del aceite de palma se encuentra en las posiciones sn1 y sn-3 del triacilglicerol. ${ }^{7,8}$ En general, los aceites vegetales tienen solo un 5-20\% del ácido palmítico en la posición sn-2. ${ }^{2}$

Los triacilgliceroles de la leche materna, con su ácido palmítico en la posición sn-2, son digeridos por la lipasa pancreática; ésta -por su acción sobre los carbonos 1 y 3-produce la hidrólisis de los ácidos grasos y libera ácidos grasos saturados, tanto palmítico y esteárico como sn-2-monoacilgliceroles, los cuales luego formarán micelas mezcladas con las sales biliares que serán adecuadamente absorbidas. ${ }^{4}$

Contrariamente a este camino, el ácido palmítico de los triacilgliceroles proveniente del aceite de palma en la posición sn-1 y sn-3, al ser hidrolizado, forma ácido palmítico libre. Estos ácidos grasos libres se unen al calcio en un proceso de saponificación y forman un complejo no absorbi- ble que contribuye a la baja absorción de calcio y grasas, a la menor ganancia de peso y al endurecimiento de la materia fecal. Este efecto del aceite de palma ha sido demostrado en niños de término y pretérmino, y en animales de experimentación. ${ }^{9}$ La consecuencia fisiológica es la disminución de la biodisponibilidad de calcio, que tiene un efecto negativo demostrable también en el esqueleto. ${ }^{9,10}$

La grasa láctea proveniente de leche de vaca naturalmente presenta una concentración superior al $45 \%$ de ácido palmítico en la posición sn$2 .{ }^{11}$ En el mercado también se presentan lípidos estructurados en los sustitutos de la leche materna, que alcanzan el $45 \%$ del ácido palmítico en la posición sn-2. ${ }^{12}$

El objetivo del presente trabajo es analizar el perfil de triacilgliceroles y el porcentaje de ácido palmítico en la posición sn-2 en sustitutos de la leche materna (fórmulas infantiles de uso en el primer semestre de vida) que presentan grasa láctea o aceites vegetales en su composición (o ambos).

\section{MATERIAL Y MÉTODOS}

Se analizaron 6 productos del mercado argentino que se presentan como sustitutos de la leche

TABLA 1. Composición porcentual de las fórmulas según información disponible de los fabricantes

\begin{tabular}{|c|c|c|c|c|c|}
\hline Fórmula & Grasa láctea $(\%)$ & Aceites (\%) & Lípidos estructurados (\%) & Aceite de pescado (\%) & Otros (\%) \\
\hline 1 & & $\begin{array}{c}\text { Palma: } 49 \\
\text { Colza (canola): } 11 \\
\text { Maíz: } 22 \\
\text { Oleína de palma: } 17 \\
\text { AGPI*: } 1\end{array}$ & & & \\
\hline 2 & & $\begin{array}{c}\text { Palma: } 40 \\
\text { Coco: } 22 \\
\text { Colza (canola): } 20 \\
\text { Maíz: } 18\end{array}$ & & & \\
\hline 3 & 68 & $\begin{array}{l}\text { Colza (canola): 15,8 } \\
\text { Girasol: 15,8 }\end{array}$ & & & Células simples: 0,4 \\
\hline 4 & & $\begin{array}{c}\text { Colza (canola): } 12 \\
\text { Coco: } 9 \\
\text { Girasol: } 9\end{array}$ & 67 & 1 & $\begin{array}{l}\text { Lecitina de soja: } 1 \\
\text { Células simples: } 1,5\end{array}$ \\
\hline 5 & 69 & $\begin{array}{l}\text { Colza (canola): 13,6 } \\
\text { Girasol: 13,6 }\end{array}$ & & 1,6 & $\begin{array}{l}\text { Células simples: } 1,5 \\
\text { Lecitina de soja: } 0,7\end{array}$ \\
\hline 6 & & $\begin{array}{c}\text { Girasol: } 20 \\
\text { Coco: } 20 \\
\text { Colza (canola): } 14 \\
\text { Palma: } 40 \\
\text { Prímula (onagra): } 5\end{array}$ & & & $\begin{array}{c}\text { Células simples, } \\
\text { aceite de pescado } \\
\text { y } \mathrm{TCM}^{* *}: 1\end{array}$ \\
\hline
\end{tabular}

*AGPI: Ácidos grasos poliinsaturados de cadena larga.

**TCM: triglicéridos de cadena media.

Canola: denominación comercial del aceite de colza (Brassica napus) (acrónimo de Canadian oil low acid). 
materna durante el primer semestre de vida, a saber:

- 2 fórmulas con aceites vegetales como fuente básica de lípidos (fórmulas 1 y 2);

- 1 fórmula con grasa láctea como base y aceites vegetales (fórmula 3);

- 1 fórmula con lípidos estructurados y aceites vegetales (fórmula 4);

- 1 fórmula para prematuros con grasa láctea como base y otros aceites (fórmula 5);

- 1 fórmula para prematuros con aceites vegetales como base (fórmula 6).

Las fórmulas fueron adquiridas en farmacias de la Argentina y analizadas antes de la fecha de expiración para su uso.

La composición porcentual de las fórmulas analizadas, según información disponible de los fabricantes, se observa en la Tabla 1. La presencia de aceite de tipo "células simples" se refiere a ácido docosahexaenoico (ADH) y ácido araquidónico (ARA), producidos a partir de la fermentación del aceite y harina de soja con el hongo Mortierella alpina.

\section{Análisis de ácidos grasos}

Extracción de lípidos totales: Los lípidos fueron extraídos con ciclohexano, según método de Monteiro-Riviere y cols. ${ }^{13}$

Hidrólisis de acilgliceroles: Se utilizó lipasa pancreática con aislamiento posterior de los productos hidrolíticos por TLC, según técnica de Luddy y cols. ${ }^{14}$

Formación de metilésteres de ácidos grasos: Los ácidos grasos obtenidos fueron metilados con una mezcla de trifloruro de boro-metanol según método ISO 5509:2000. Los metilésteres fueron luego cromatografiados mediante cromatografía gaseosa (cromatógrafo gaseoso, Agilent Technologies). Los análisis se realizaron en Escocia, en Mylnefield Research Services Ltd., miembro del Scottish Crop Research Institute Group.

\section{RESULTADOS}

En la Tabla 1 se observa que el aceite de palma está presente en un alto porcentaje en las fórmulas 1 y 2 ( $40 \%$ y $49 \%$, respectivamente), diseñadas para lactantes de término en el primer semestre de vida, y en la fórmula $6(40 \%)$ para recién nacidos pretérmino, que no contienen grasa láctea o lípidos estructurados. La colza (canola) está presente en todas las fórmulas, con un intervalo entre $11 \%$ y $20 \%$, asociada al maíz en las fórmulas 1 y 2 , o al girasol en las cuatro fórmulas restantes. El aceite de coco está presente en las fórmulas 1, 2, 4 y 6.

En la Tabla 2 se describe la concentración final de ácido palmítico en la posición sn-2 de cada fórmula y la relación entre esta posición y las posiciones sn-1 y sn-3.

Las dos fórmulas con base láctea (3 y 5) presentan la mayor concentración de ácido palmítico en la posición sn-2 (48,1\% y 49,5\%, respectivamente), mientras que la fórmula con lípidos estructurados presenta $44,5 \%$ en esa posición.

Otra forma de expresar la distribución de los ácidos grasos que esterifican al glicerol es a través de la relación de aquellos ubicados en la posición sn-2 sobre la suma de los ubicados en las posiciones sn-1 y sn-3. De esta manera, la relación en las fórmulas que contienen ácido palmítico en la posición sn-2 es alta (entre 1,6 y 2).

\section{DISCUSIÓN}

Los lípidos de los sustitutos de la leche materna están compuestos por diferentes tipos de aceites en diferentes concentraciones. Muchas de las fórmulas estudiadas también se comercializan en otros mercados del mundo, con las mismas concentraciones de lípidos halladas en nuestro estudio. ${ }^{12}$

La fórmula que logra la mayor aproximación de concentración de ácido palmítico en la posición sn-2 respecto de la leche materna es la que está basada en grasa láctea y complementa su perfil lipí-

TABLA 2. Productos y porcentaje final de ácido palmítico en la posición sn-2

\begin{tabular}{llcc}
\hline Fórmula & Producto & \% final de ácido palmítico en posición sn-2 & Relación sn-2:sn1+sn-3 \\
\hline 1 & Base de aceites vegetales $1\left(1^{\text {er. }}\right.$ semestre $)$ & $14,6 \%$ & $14,8 \%$ \\
2 & Base de aceites vegetales $2\left(1^{\text {er. }}\right.$ semestre $)$ & $48,1 \%$ & 0,4 \\
3 & Base de grasa láctea $\left(1^{\text {er. }}\right.$ semestre $)$ & $44,5 \%$ & 1,8 \\
4 & Base de lípidos estructurados $\left(1^{\text {er. }}\right.$ semestre $)$ & $49,5 \%$ & 1,6 \\
5 & Base de grasa láctea para prematuros & $14,6 \%$ \\
6 & Base de aceites vegetales para prematuros & 2 \\
\hline
\end{tabular}


dico con aceites vegetales (colza y girasol). En el caso de la fórmula para prematuros, la de mayor aproximación es también la que está basada en grasa láctea y completa su formulación con aceite vegetal y aceite de pescado. También logra una buena aproximación la fórmula con lípidos estructurados, como fue descrito por otros autores. ${ }^{12}$

Las fórmulas que contienen básicamente aceites vegetales presentan los porcentajes más bajos de ácido palmítico en la posición sn-2, como ya ha sido descrito. ${ }^{2}$

Las publicaciones acerca de la presencia de ácido palmítico y su distribución posicional en las fórmulas infantiles no remarcan la diferencia entre las fórmulas que contienen aceites vegetales y las que contienen grasa láctea. ${ }^{2}$

La distribución de ácido palmítico en los triacilgliceroles tiene especial interés, ya que en estudios con preparados para lactantes ricos en ácido palmítico esterificado en la posición sn-2 de los triacilgliceroles, los niveles de absorción de ácido palmítico fueron más altos que cuando el compuesto se ubica principalmente en las posiciones sn-1 y sn-3., ${ }^{410}$

La leche materna es la mejor referencia para la alimentación del lactante. Presenta la mayor concentración de ácido palmítico en la posición sn-2. Evidentemente, en esta etapa de la vida, el ácido palmítico en esa posición de los triacilgliceroles constituye una ventaja, atendiendo a la mejor absorción de grasa (etapa crítica del desarrollo del SNC) y calcio (velocidad de crecimiento y acreción de calcio) antes mencionada. ${ }^{9,10}$ Uno de los principales lípidos estructurales de las membranas del tejido nervioso es la esfingomielina, y el ácido palmítico es uno de los principales ácidos grasos constituyente de estos esfingolípidos. ${ }^{15}$

El ácido palmítico es una grasa saturada; en el niño mayor de 2 años y en el adulto, el aporte sostenido de grasas saturadas, como el palmítico, se asocia con mayor riesgo de enfermedad cardiovascular a través de un aumento de la colesterolemia.

Las fórmulas infantiles para lactantes de término y pretérmino analizadas en este estudio y que contienen grasa láctea presentaron el mayor porcentaje de ácido palmítico en la posición sn-2.

Se requieren más estudios para determinar si las diferencias químicas de los productos analizados se corresponden con diferentes manifestaciones clínicas.

\section{Agradecimientos}

A los bioquímicos escoceses G. Sawers y S.A. Rowbotton que realizaron las determinaciones en el laboratorio de Mylnefield Research Services Ltd., y a Adriana Di Maggio por la edición del trabajo y la traducción del resumen.

\section{BIBLIOGRAFÍA}

1. Koletzko B, Lien E, Agostoni C, Bo H, et al. The roles of long-chain polyunsaturated fatty acids in pregnancy, lactation and infancy: review of current knowledge and consensus recommendations. J Perinat Med 2008;36:5-14.

2. Straarup EM, Lauritzen L, Faerk J, Hoy CE, Michaelsen KF. The stereospecific triacylglycerol structures and fatty acid profiles of human milk and infant formulas. J Pediatr Gastroenterol Nutr 2006;42:293-9.

3. Lien EL. The role of fatty acid composition and positional distribution in fat absorption in infants. J Pediatr 1994;125:62-8.

4. López-López A, López-Sabater MC, Campoy-Folgoso C, M Rivero-Urgell M, Castellote-Bargallo AI. Fatty acid and sn-2 fatty acid composition in human milk from Granada (Spain) and in infant formulas. Eur J Clin Nutr 2002;56: 1242-54.

5. Innis SM. Human milk and formula fatty acids. J Pediatr 1992;120:S56-61.

6. Koletzko B, Thiel I, Abiodun PO. The fatty acid composition of human milk in Europe and Africa. J Pediatr 1992;120: S62-70.

7. Nelson C, Innis S. Plasma lipoprotein fatty acids are altered by the positional distribution of fatty acids in infant formula triacylglycerols and human milk. Am J Clin Nutr 1999;70:62-9.

8. Carnielli VP, Luijendijk IHT, van Goudoever JB, Sulkers EJ, et al. Structural position and amount of palmitic acid in infant formulas: effects on fat, fatty acid and mineral balance. J Pediatr Gastroenterol Nutr 1996;23:553-60.

9. Koo WW, Hammami M, Margeson DP, Nwaesei C, et al. Reduced bone mineralization in infants fed palm oleincontaining formula: a randomized, double-blinded, prospective trial. Pediatrics 2003;111:1017-23.

10. Koo WW, Hockman EM, Dow M. Palm olein in the fat blend of infant formulas: effect on the intestinal absorption of calcium and fat, and bone mineralization. J Am Coll Nutr 2006;25:117-22.

11. Freeman CP, Jack EL, Smith LM. Intramolecular fatty acid distribution in the milk fat triglycerides of several species. J Dairy Sci 1965;48:853-8.

12. Lucas A, Quinlan P, Abrams S, Ryan SS, et al. Randomised controlled trial of a synthetic triglyceride milk formula for preterm infants. Arch Dis Child 1997;77(3):F178-84.

13. Monteiro-Riviere NA, Inman AO, Mak V, Wertz P, Riviere JE. Effect of selective lipid extraction from different body regions on epidermal barrier function. Pharm Res 1984;18:992-8.

14. Luddy FE, Barford RA, Herb SF, Magidman P, Riemenschneider RW. Pancreatic lipase hydrolysis of triglicerides by a semi-microtechnique. J Am Oil Chemist's Society 1964;41:693-6.

15. Hawthorne JN, Ansell, GB. Phospholipids: New Comprehensive Biochemistry, vol. 4 (Neuberger A, van Deenen LLM, series eds). Amsterdam: Elsevier Biomedical Press; 1982. 